\title{
Computational Engineering Modeling for Runner Athletes
}

\author{
Soheila Yavarmasroor, ${ }^{1}$ Zahra Hojjati Zidashti, ${ }^{2}$ \\ Akbar Khodaparast Haghi, ${ }^{3}$ and Kaveh Hariri Asli ${ }^{4}$ \\ ${ }^{1}$ Science and Research Branch, Islamic Azad University of Iran, No. 1045, Alley Azerbany2, Farhang ring, Rasht, Gilan, Iran \\ ${ }^{2}$ Islamic Azad University of Iran, Rasht Branch, Gilan, Iran \\ ${ }^{3}$ University of Guilan, Rasht, Iran \\ ${ }^{4}$ National Academy of Science of Azerbaijan, Baku, Azerbaijan
}

Correspondence should be addressed to Soheila Yavarmasroor; soheila.yavarmasroor@yahoo.com

Received 16 April 2013; Revised 13 July 2013; Accepted 28 July 2013

Academic Editor: Jia-Jang Wu

Copyright (C) 2013 Soheila Yavarmasroor et al. This is an open access article distributed under the Creative Commons Attribution License, which permits unrestricted use, distribution, and reproduction in any medium, provided the original work is properly cited.

The literature indicates that relatively little research is available to describe the relationship between functional running tasks and characteristics of individuals who perform these tasks. As a main purpose, the present work is to define the computational modeling for anthropometric characteristics of athletes. Thus the dynamic model presented by this 100-meter running test can play an important role in talent and coaching. The research question was formed by classification and comparison of statures of sportswomen with other anthropometric classes. On the other hand, the present work compares the anthropometric data for runner velocity (running time) against runner weight. The method of research is regression statistical analysis method. In this work, the regression method is based on the univariable ANOVA variance with repeated measures and $t$-test for independent samples. Data analysis was performed by using the software SPSS13. The results of the 100-meter running test of sportswomen showed good correlation between the parameters. As a dynamic modeling selection, the logarithmic function showed suitable correlation on scatter diagram. Consequently, the results of this work will help to reduce the risk of sportswomen activities. Therefore it can be recommended for medical professionals and athletic talent.

\section{Introduction}

Historical anthropometrics is the study of patterns in human body size and their correlates over time. While social researchers, public health specialists, and physical anthropologists have long utilized anthropometric measures as indicators of well being, only within the past three decades have historians begun to use such data extensively. Adult stature is a cumulative indicator of net nutritional status over the growth year and thus reflects command over food and access to healthful surroundings. Since expenditures for these items comprised such a high percentage of family income for historical communities, mean stature can be used to examine changes in a population's economic circumstances over time and to compare the well being of different groups with similar genetic height potential. Anthropometric measures are available for portions of many national populations as far back as the early 1700s. While these data often serve as complements to standard economic indicators, in some cases, they provide the only means of assessing historical economic well being, as "conventional" measures such as per capita GDP, wage, and price indices, and income inequality measures have been notoriously spotty and problematic to develop. Anthropometric-based research findings to date have contributed to the scholarly debates over mortality trends, the nature of slavery, and the outcomes of industrialization and economic development. Height has been the primary indicator utilized to date. Other indicators include height-standardized weight indices, birth weight, and age at menarche. Potentially even more important, historical anthropometrics broadens the understanding of "well being" beyond the one-dimensional "ruler" of income, providing another lens through which the quality of historical life can be viewed. Clinical anthropometric experience suggests that many clinicians incorporate progressive squat running exercises in interventions for patients who have incurred 
such an injury and who wish to return to work that will require them to continue performing this type of running task. Clinicians, therefore, often may need to advise their patient and the patient's employer regarding the patient's ability to return to full or restricted work. A patient's ability to return to work may be assessed by several different strategies. One approach might be to evaluate the job requirements and determine whether the job poses risks for injury regardless of the patient's rehabilitation status. The National Institute for Occupational Safety and Health (NIOSH) sponsored a panel of experts in 1985 who used existing research as a basis for recommending criteria to determine safe running range. The intent of the NIOSH running equations that evolved from this meeting was to identify running tasks that posed risk for the development of running-related injuries and to alter these job tasks to reduce the risks of injury. The NIOSH equations were revised in 1991 to include new information and address additional aspects of running safety that included issues such as asymmetric running, different hand container couplings, work duration, and running frequency. These equations are based on biomechanical, physiological, and psychosocial criteria related to running tasks. Unfortunately, the equations are geared solely to the running tasks and assumed population parameter and do not address the ability of a specific individual to perform the running tasks safely. The results of a recent cross-validation study also call into question the validity of the biomechanical and physiological criteria used in the 1991 equations. Decisions regarding ability to return to work also may be based on the individual's ability to perform the tasks required by the job. Clinicians could attempt to simulate lifting tasks required by the job and determine when the patient could safely lift these loads. Several issues may exist for this particular strategy. Clinicians may not be able to replicate in the clinic all of the running-related variables that exist at the work site. Some of these variables include duration of the workday, frequency of running, and environmental conditions (e.g., ambient temperature). Also, even if patients have completed a comprehensive rehabilitation program, they may not be capable of safely performing the running requirements for the particular job to which they wish to return. The ability to estimate a patient's preinjury running ability relative to the job's running requirements might enable clinicians to determine whether the job in question poses undue risk of injury for the patient. A hypothetical scenario might involve a patient's preinjury running capacity being less than the running requirements for the very job that the patient was performing at the time of injury. Such a determination might indicate that the patient should pursue job reassignment or new employment at the beginning of a course of rehabilitation. Postinjury determination of running range might then confirm the previous estimate of running range. The initial estimate of running range, however, might be valuable in counseling the patient and the employer early in rehabilitation regarding more appropriate job assignment. Another issue also relates to knowledge of a patient's preinjury running ability. Clinicians might determine that a patient can safely running. The patient, however, may still not have progressed in his or her rehabilitation program to a point that approximates the patient's preinjury running range.
Patients in this situation might be discharged prematurely from therapy and might be at risk for injury for lift tasks that they typically had performed prior to injury at sites other than work (e.g., home). The ability to estimate running range might also be useful in screening potential employees for manual-running jobs without involving the time, training, and potential risk of actually testing their running range. Another approach that has been taken to estimate maximum running range for an individual involves using multiple test variables from an instrumented treadmill to model performance on a functional running task. A principal components analysis of the 32 incremental treadmill test variables yielded four factors that explained $89.2 \%$ of the variance of treadmill performance: maximum strength, midbody coordination, minimum strength, and lower body coordination. The limitations of this particular strategy are that instrumentation such as the incremental lifting machine is expensive, and the incremental treadmill testing protocol involves having an individual perform multiple running, which may be time consuming and may pose some risk for injury of the individual being tested. The literature indicates that relatively little research is available to describe the relationship between functional lifting tasks and characteristics of individuals who perform these tasks. Considerable research does exist, however, indicating significant relationships between the strength of specific muscle groups and anthropometric and demographic variables. Gross et al. have provided a review of much of this literature. Some of these previous studies involve an analysis of simple correlations between muscle strength and specific predictor variable. Several studies, however, indicate the value of using multiple variables in regression analyses to predict normal strength. The results of these latter studies raise the question of whether a similar approach might be useful in predicting normal functional running range on the basis of models that include multiple anthropometric and demographic predictor variables [1-4].

\section{Materials and Methods}

The purpose of this study, therefore, was to determine the relationship between runner velocity and anthropometric variables. Variables were selected for the study on the basis of theoretical modeling or previous research regarding the relationship between study variables and runner velocity range. The specific research question addressed was whether a significant relationship exists between runner velocity range and some combination of the following variables: age, height, weight, stature, percentage of body fat, arm length, thigh girth, calf girth, pelvic width, and pelvic girth. In this work, dateline for field tests data collection was: at 09:00 a.m., 10/09/2012 until 27/09/2012. Location of work field tests model was at Rasht city in the north of Iran. In this work, the following considerations were made when using and applying anthropometric data for heart rate of runner related to runner velocity (running time) and runner weight. Heart rate of runner was measured in two cases: first at the running state (dynamic heart rate) and second before running (static heart rate). Twenty healthy and young sprint runner females (Tables 1 and 2) participate in this study. They 
TABLE 1: Field tests and anthropometric model for velocity.

\begin{tabular}{|c|c|c|c|c|c|}
\hline No. & Age & Running time (sec) & Running speed $(\mathrm{m} / \mathrm{sec})$ & Hip circumference & Waist circumference \\
\hline 1 & 19 & 13.45 & 7.43 & 90 & 64 \\
\hline 2 & 16 & 13.66 & 7.32 & 94 & 71 \\
\hline 3 & 18 & 15.03 & 6.65 & 88 & 65 \\
\hline 4 & 16 & 13.67 & 7.31 & 91 & 68 \\
\hline 5 & 14 & 14.30 & 6.99 & 92 & 66 \\
\hline 6 & 14 & 14.73 & 6.78 & 82 & 63 \\
\hline 7 & 14 & 17.10 & 5.84 & 93 & 66 \\
\hline 8 & 20 & 17.55 & 5.69 & 88 & 65 \\
\hline 9 & 21 & 16.28 & 6.14 & 92 & 69 \\
\hline 10 & 14 & 16.03 & 6.23 & 92 & 70 \\
\hline 11 & 18 & 16.27 & 6.14 & 94 & 70 \\
\hline 12 & 15 & 16.77 & 5.96 & 98 & 67 \\
\hline 13 & 23 & 17.35 & 5.76 & 88 & 68 \\
\hline 14 & 22 & 15.22 & 6.57 & 90 & 64 \\
\hline 15 & 21 & 14.98 & 6.67 & 95 & 69 \\
\hline 16 & 21 & 14.90 & 6.71 & 98 & 73 \\
\hline 17 & 22 & 14.51 & 6.89 & 97 & 71 \\
\hline 18 & 28 & 13.98 & 7.15 & 95 & 70 \\
\hline 19 & 22 & 14.45 & 6.92 & 92 & 66 \\
\hline 20 & 24 & 16.98 & 5.88 & 97 & 78 \\
\hline
\end{tabular}

TABLE 2: Regression anthropometric model for velocity.

\begin{tabular}{|c|c|c|c|c|c|c|c|}
\hline No. & Age & Stature $(\mathrm{cm})$ & Weight $(\mathrm{kg})$ & BMI & WHR & Dynamic heart rate & Static heart rate \\
\hline 1 & 19 & 165 & 50 & 18.38 & 0.71 & 176 & 74 \\
\hline 2 & 16 & 159 & 52 & 20.63 & 0.75 & 173 & 72 \\
\hline 3 & 18 & 168 & 47 & 16.66 & 0.73 & 174 & 70 \\
\hline 4 & 16 & 158 & 50 & 20.08 & 0.74 & 111 & 70 \\
\hline 5 & 14 & 164 & 52 & 19.44 & 0.71 & 173 & 79 \\
\hline 6 & 14 & 166 & 44 & 16 & 0.76 & 178 & 77 \\
\hline 7 & 14 & 161 & 54 & 20.84 & 0.7 & 191 & 111 \\
\hline 8 & 20 & 161 & 46 & 17.76 & 0.73 & 133 & 83 \\
\hline 9 & 21 & 159 & 48 & 19.04 & 0.75 & 160 & 82 \\
\hline 10 & 14 & 167 & 52 & 18.7 & 0.76 & 150 & 79 \\
\hline 11 & 18 & 168 & 56 & 19.85 & 0.74 & 153 & 75 \\
\hline 12 & 15 & 64 & 55 & 20.52 & 0.68 & 166 & 81 \\
\hline 13 & 23 & 154 & 45 & 18.98 & 0.77 & 140 & 94 \\
\hline 14 & 22 & 170 & 56 & 19.37 & 0.71 & 154 & 73 \\
\hline 15 & 21 & 174 & 59 & 19.53 & 0.72 & 164 & 79 \\
\hline 16 & 21 & 151 & 55 & 24.12 & 0.74 & 177 & 77 \\
\hline 17 & 22 & 170 & 62 & 21.45 & 0.73 & 93 & 75 \\
\hline 18 & 28 & 158 & 55 & 22.08 & 0.73 & 170 & 68 \\
\hline 19 & 22 & 165 & 56 & 20.58 & 0.71 & 166 & 70 \\
\hline 20 & 24 & 166 & 62 & 22.54 & 0.8 & 168 & 83 \\
\hline
\end{tabular}

signed a written/informed consent. All subjects completed a medical questionnaire to ensure that they were not taking any medication and were free from cardiac, respiratory, renal, or metabolic diseases. They did not have any orthopaedic injury that would inhibit physical activity. The subjects were tested in the follicular phase of the menstrual cycle based on their previous menses. All participants consumed their last meal at least $2 \mathrm{~h}$ before the test and refrained from intensive exercise in the $24 \mathrm{~h}$ period preceding testing [5-10]. All anthropometric measures were recorded in the morning by the same experienced anthropometrist. The weight was measured using a wall-mounted stadiometer $( \pm 0.1 \mathrm{~cm})$. The exercise systolic and diastolic heart rates were measured on the left arm of each participant using cuffs of appropriate size (Table 1). 
The measurements were recorded just after $100 \mathrm{~m}$ intense running in standing position. In the present work, for professional runner at fast running, heart rate of runner, runner velocity, and weight were recorded. Curve estimation procedure has used these data which have been detected field tests (Tables 1 and 2). These data have been compared with heart rate of runner and runner velocity which have been collected from actual systems (field tests). The model was calibrated using one set of data, without changing parameter values. It was used to match a different set of results. Regression model (Table 3 ) has been built based on field tests data [11-14].

\section{Results and Discussion}

In this work, conclusions were drawn on the basis of experiments and calculations for independent variables (running weight) based on the

$$
V=f(W)
$$

dependent variable: $V$-runner velocity (running time), with nomenclature " $Y$ " for first: at the running state and the second: before running condition.

Independent variable with nomenclature " $X$ " such as $W$-runner weight $(\mathrm{kg})$ is the most important variable.

The most important effects that were observed based on regression for model summary (Table 2) are as follows (Figure 1). During dynamic exercise, it is generally assumed that heart rate increases due to both a parasympathetic withdrawal and an augmented sympathetic activity. In Figure 1, there is a statement on methods of nonlinear dynamics. Methods of nonlinear dynamics define parameters that quantify complicated interactions of independent and interrelated components, which can be described as complexity measures. Methods related to the chaos theory are used to describe the nonlinear properties of heart rate fluctuations. Dependent variable includes runner velocity or running time and the independent variable includes runner weight which is the most important variable. The most important effects that were observed based on regression for model summary were shown in Figure 1. During dynamic exercise, it is generally assumed that heart rate increases due to both a parasympathetic withdrawal and an augmented sympathetic activity.

There is also a technical problem related to the nonstationary signals; a critical look at interpretation of results is needed. It is strongly suggested that, when presenting reports on HRV studies related to exercise physiology in general or concerned with athletes, a detailed description should be provided on analysis methods, as well as concerning population, and training schedule, intensity, and duration. Most studies concern relatively small numbers of study participants, diminishing the power of statistics $[15,16]$.

Thus, the present work obtains the following model to approximate the variables.

\section{Linear Function}

$$
y=16.41-.021 x
$$

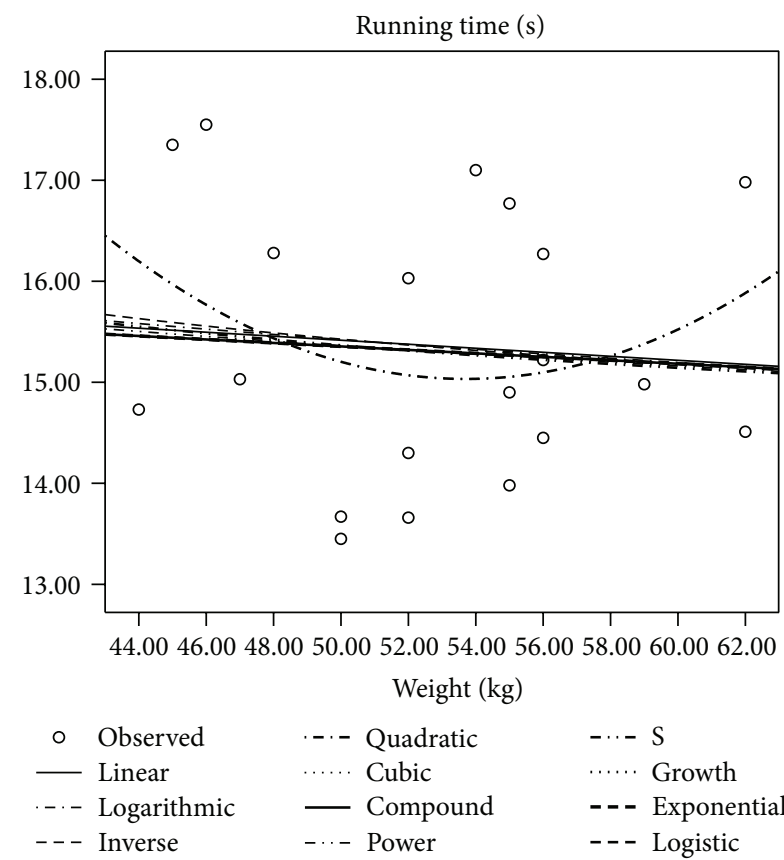

FIGURE 1: Curve fit for running time against weight.

Logarithmic Function

$\log y=\log (-1.242)+(20.281) \log x \quad$ or $\quad y=.062 x^{1.001}$.

Compound Function

$$
A=16.24 e^{.999 t} \text {. }
$$

Quadratic Function

$$
y=50.686-(1.328) x+(.012) x^{2}
$$

Growth Function

$$
\left(\frac{d A}{d T}\right)=K A .
$$

Exponential Function

$$
y=16.24(-.001)^{x}+g
$$

Logistic Function

$$
y=.062 x^{1.001}+g
$$

\section{Cubic Function}

$$
y=50.686+1.328 x+.012 x^{2}+o x^{3} .
$$

These functions were built based on field tests data. As a dynamic modeling selection, the logarithmic function had suitable correlation on scatter diagram (Figure 1). Therefore it was selected as the regression model for this work. The results of the present work were compared with the works of other experts. 
TABLE 3: Model summary and parameter estimates for running time.

\begin{tabular}{|c|c|c|c|c|c|c|c|c|c|}
\hline \multirow[t]{2}{*}{ Equation } & \multicolumn{5}{|c|}{ Model summary } & \multicolumn{4}{|c|}{ Parameter estimates } \\
\hline & $R$ square & $F$ & $\mathrm{df1}$ & $\mathrm{df} 2$ & Sig. & Constant & $b 1$ & $b 2$ & $b 3$ \\
\hline $\begin{array}{l}\text { Linear equation } \\
y=a_{0}+a_{1} x\end{array}$ & 0.006 & 0.110 & 1 & 18 & 0.744 & 16.410 & -0.020 & & \\
\hline $\begin{array}{l}\text { Quadratic } \\
y=a_{0}+a_{1} x+a_{2} x^{2}\end{array}$ & 0.082 & 0.761 & 2 & 17 & 0.482 & 50.686 & -1.328 & 0.012 & \\
\hline $\begin{array}{l}\text { Cubic } \\
Y=a_{0}+a_{1} x+a_{2} x^{2}+a_{3} x^{3}\end{array}$ & 0.082 & 0.761 & 2 & 17 & 0.482 & 50.686 & -1.328 & 0.012 & 0.00 \\
\hline $\begin{array}{l}\text { Compound } \\
A=c e^{k t}\end{array}$ & 0.005 & 0.083 & 1 & 18 & 0.776 & 16.240 & 0.999 & & \\
\hline $\begin{array}{l}\text { Growth } \\
A=c e^{k t}\end{array}$ & 0.005 & 0.083 & 1 & 18 & 0.776 & 2.787 & -0.001 & & \\
\hline $\begin{array}{l}\text { Exponential } \\
y=a b^{x}+g\end{array}$ & 0.005 & 0.083 & 1 & 18 & 0.776 & 16.240 & -0.001 & & \\
\hline $\begin{array}{l}\text { Logistic } \\
y=a x^{b}+g\end{array}$ & 0.005 & 0.083 & 1 & 18 & 0.776 & 0.062 & 1.001 & & \\
\hline $\begin{array}{l}\text { Logarithmic } \\
\log y=\log a+b \log x\end{array}$ & 0.009 & 0.156 & 1 & 18 & 0.697 & 20.281 & -1.242 & & \\
\hline
\end{tabular}

$a$ : the independent variable $(\mathrm{m} / \mathrm{s})$ contains nonpositive values. The minimum value is 0.00 . The logarithmic and power models cannot be calculated. $b$ : the independent variable $(\mathrm{m} / \mathrm{s})$ contains values of zero. The inverse and S models cannot be calculated. Regression equation defined in stages $(2-3-7-8)$ has been meaningless and stages (1-4-5-6-9-10-11) have been accepted, because the coefficients are meaningful.

3.1. Comparison with Other Works. The work was compared with other experts (Rahmani-Nia et al.). Other experts obtained the effect of leptin, heart disease, and exercise. Comparison showed similarity between the present work and the results of these works. In both works the use of the new methods such as nonlinear dynamics for analysis of the nonlinear properties of fluctuations for anthropometric characteristics may provide a more sensitive way to characterize function or dysfunction of the control mechanism of the physiological variables fluctuations system. These tools are promising with regard to the understanding of the latter mechanism but are still under development and evaluation. Moreover, these methods require more powerful computing and high speed measuring instruments with up to second time responses. Nonlinear dynamical methods have made their appearance in the analysis of heart rate variability (HRV) only recently and methods have still to be established. Methods related to the chaos theory are used to describe the nonlinear properties of heart rate fluctuations. The use of the new methods from nonlinear dynamics for HRV analysis may provide a more sensitive way to characterize function or dysfunction of the control mechanism of the cardiovascular system. These tools are promising with regard to the understanding of the latter mechanism but are still under development and evaluation. Moreover, these methods require more powerful computing and are less visually attractive compared with frequency analysis in other works $[17,18]$.

\section{Conclusions}

This work showed the effects of the runner velocity as the most important variable against runner weight. The present work emphasized that "regression model" is an accurate model for modeling anthropometric characteristics of elite sportswomen and body motion. Hence in order to present the influence of runner weight on runner velocity, it was compared with the models for computational and field tests experiments. The anthropometric of body motion was defined based on these procedures. Therefore the dynamic or running states were compared at static or before running condition.

On the other hand, this idea included the proper analysis to provide a dynamic response to the shortcomings of the body motion. Because increased activity has been associated with a reduction in the risk of sudden cardiac death, this work has demonstrated that a delayed decrease in HR after exercise would be a powerful and independent predictor of all-cause mortality in patients or in general population. Consequently, the results of this work will help to reduce the risk of anthropometrics in female sports women activities.

\section{Nomenclature}

$t$ : Running time $(\mathrm{s})$

$V: \operatorname{Velocity}(\mathrm{m} / \mathrm{s})$

$W$ : Runner weight (kg).

\section{Acknowledgments}

The authors thank all specialists for their valuable observations and advice and the referees for recommendations that improved the quality of this paper.

\section{References}

[1] J. Baten, "Economic development and the distribution of nutritional resources in Bavaria, 1797-1839," Journal of Income Distribution, vol. 9, no. 1, pp. 89-106, 2000. 
[2] Z. Hojjati Zidashti, S. Yavarmasroor, and K. Hariri Asli, Computational Modeling for Anthropometry, Apple Academic Press, Inc., Exclusive worldwide distribution by CRC Press, a Taylor \& Francis Group, Toronto, Canada, 2013.

[3] D. Hurst Thomas, Skull Wars Kennewick Man, Archaeology and the Battle for Native American Identity, 2001.

[4] S. Engerman, "The height of U.S. Slaves," Local Population Studies, vol. 16, pp. 45-50, 1976.

[5] R. W. Fogel, "Economic growth, population theory, and physiology: the bearing of long-term processes on the making of economic policy," American Economic Review, vol. 84, no. 3, pp. 369-395, 1994.

[6] R. S. Goonetilleke, E. Cheuk Fan Ho, and R. H. Y. So, "Foot anthropometry in Hong Kong," in Proceedings of the ASEAN Quality Assurance Agencies Roundtable Meeting (ASEAN '97), pp. 81-88, Kuala Lumpur, Malaysia, 1997.

[7] R. A. Margo and R. H. Steckel, "The heights of American slaves," Social Science History, vol. 6, no. 4, pp. 516-538, 1982.

[8] H. E. Collin-Schramm, R. A. Kittles, D. J. Operario et al., "Markers that discriminate between European and African ancestry show limited variation within Africa," Human Genetics, vol. 111, no. 6, pp. 566-569, 2002.

[9] J. Komlos and M. Baur, "From the tallest to (one of) the fattest: the enigmatic fate of the American population in the 20th century," Economics \& Human Biology, vol. 2, no. 1, pp. 57-74, 2004.

[10] J. Komlos and P. Kriwy, "The biological standard of living in the two Germanies," German Economic Review, vol. 4, no. 4, pp. 459-473, 2003.

[11] F. Rahmani-Nia and Z. Hodjati, "The effect of exercise training on body composition and aerobic power in sedentary college age females," International Journal of Fitness, India, vol. 1, pp. 24-31, 2005.

[12] F. Rahmani-Nia and Z. Hodjati, "Effect of selected training on body composition \& aerobic power of females' college," Journal of Harkat, vol. 1, pp. 18-21, 2004.

[13] F. Rahmani-Nia, N. Rahnama, Z. Hojjati, and B. Soltani, "Acute effects of aerobic and resistance exercises on serum leptin and risk factors for coronary heart disease in obese females," Sport Sciences for Health, vol. 2, no. 3, pp. 118-124, 2008.

[14] J. P. Rushton, "Race, brain size and intelligence: a rejoinder to Cain and Vanderwolf," Personality and Individual Differences, vol. 11, no. 8, pp. 785-794, 1990.

[15] M. Sunder, "The making of giants in a Welfare state: the Norwegian experience in the twentieth century," Economics \& Human Biology, vol. 1, no. 2, pp. 267-276, 2004.

[16] U. Woitek, "Height cycles in the eighteenth and nineteenth centuries," Economics \& Human Biology, vol. 1, no. 2, pp. 243-257, 2003.

[17] F. Rahmani-Nia, Z. Hojjati, N. Rahnama, and B. Soltani, "Leptin, heart disease and exercise," World Journal of Sport Sciences, vol. 2, no. 1, pp. 13-20, 2009.

[18] S. Yavarmasroor, Z. Hojjati Zidashti, and A. Khodaparast Haghi, "Dynamic modeling for sportswomen health," Sport Sciences for Health, vol. 9, no. 2, pp. 73-79, 2013. 

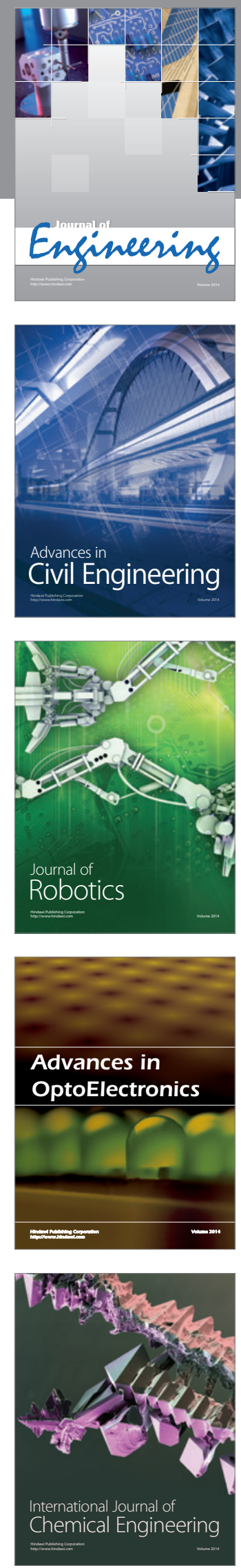

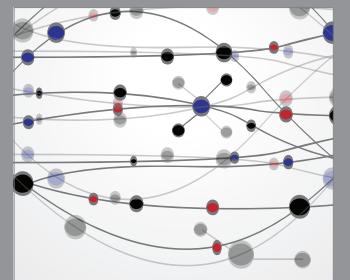

The Scientific World Journal
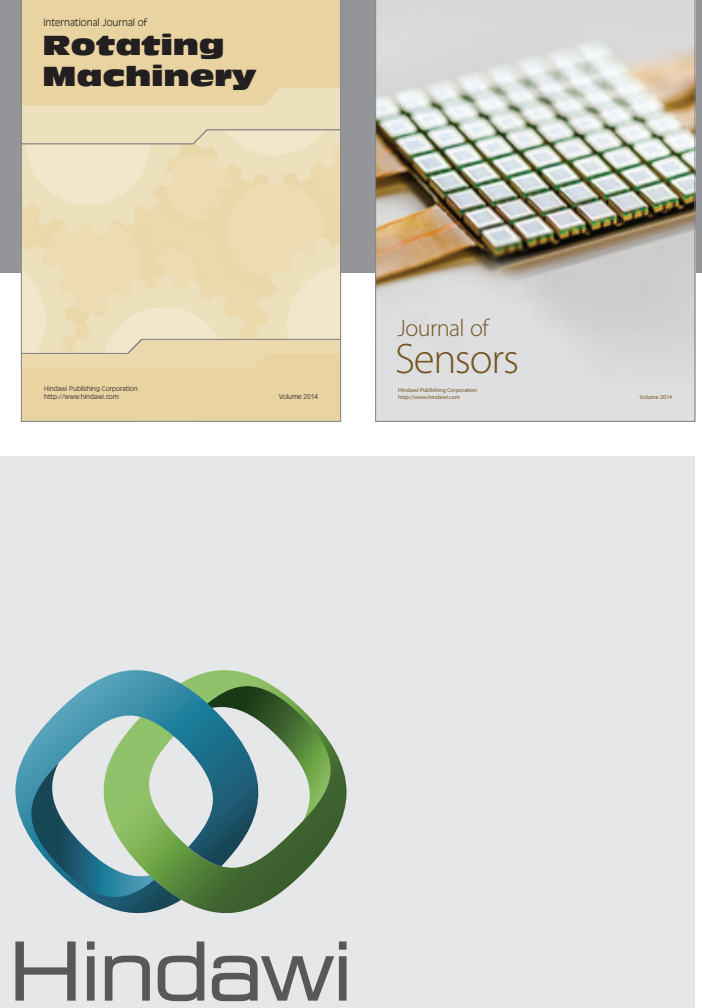

Submit your manuscripts at http://www.hindawi.com
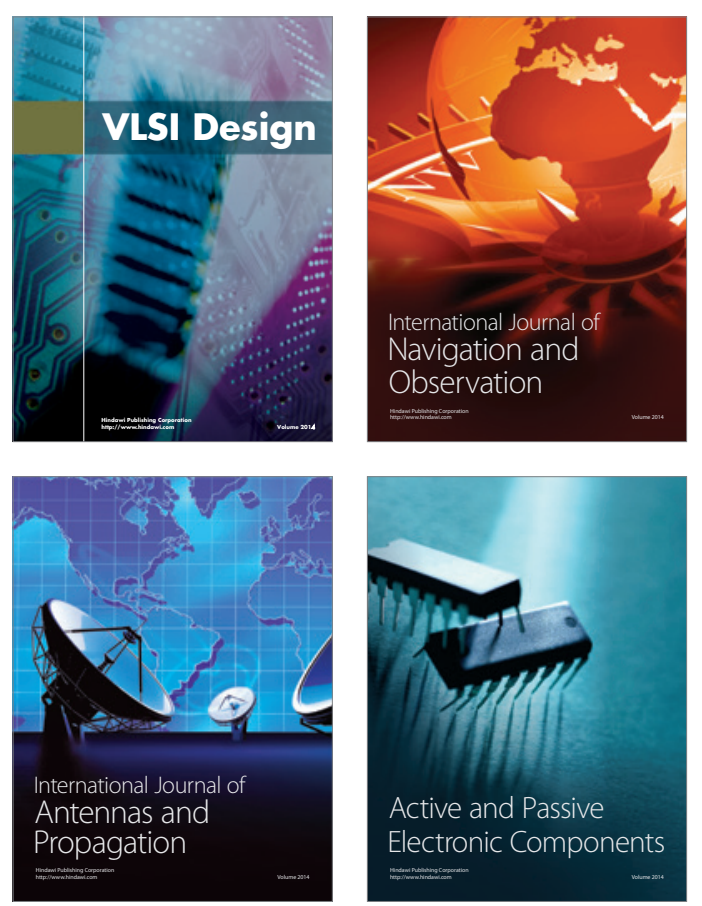
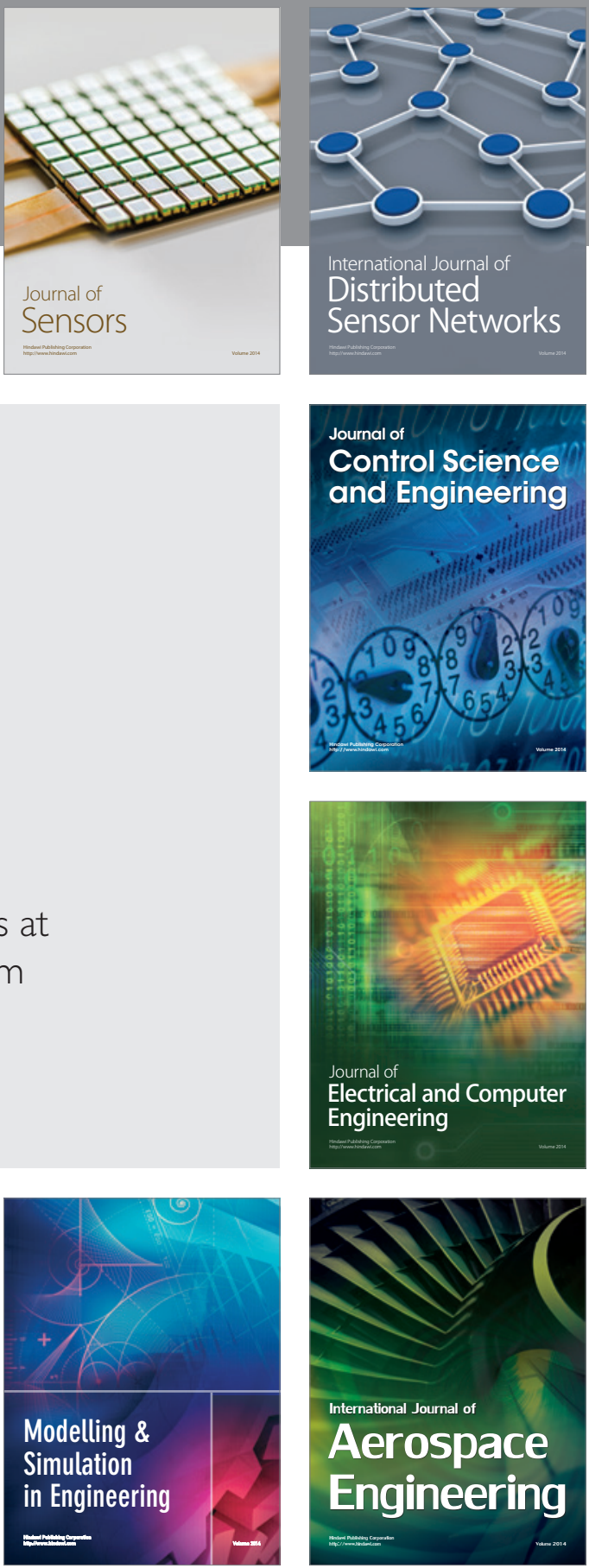

Journal of

Control Science

and Engineering
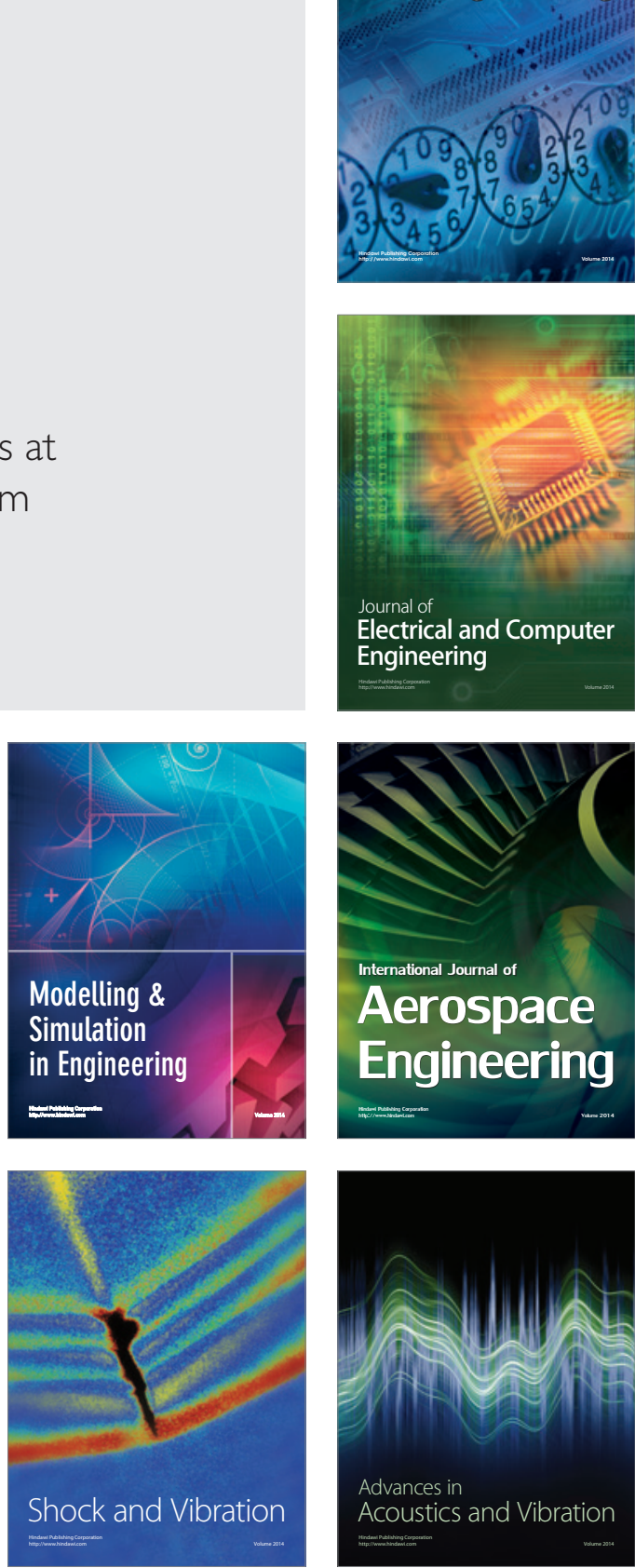